\title{
Single Organ Segmentation Filters for Multiple Organ Segmentation
}

\author{
Jacob D. Furst, Ruchaneewan Susomboom, and Daniela S. Raicu
}

\begin{abstract}
In this paper, we propose an approach for automatic organ segmentation in Computed Tomography (CT) data. The approach consists of applying multiple single organ segmentation filters and resolving conflicts among the single organ segmentations to generate a multiple organ segmentation. Each of the single organ segmentations consists of three stages: first, a probability image of the organ of interest is obtained by applying a binary classification model obtained using pixelbased texture features; second, an adaptive split-and-merge segmentation algorithm is applied on the organ probability image to remove the noise introduced by the misclassified pixels; and third, the segmented organ's boundaries from the previous stage are iteratively refined using a region growing algorithm. The conflict resolution among the single organ segmentations involves comparing region sizes and average probabilities over contested pixels.
\end{abstract}

\section{INTRODUCTION}

Automatic analysis of images from various medical imaging modalities is necessary to increase the productivity of radiologists when interpreting and diagnosing hundreds of images every day. Image segmentation is an important first step in analyzing medical data and serves as a vital preliminary step in many imaging applications such as diagnosis, anatomical structure studies, treatment planning, and computer integrated surgery [9].

In general, the approaches applied for segmentation can be divided into eight categories [5]: 1) threshold approaches, 2) region growing approaches, 3) clustering approaches, 4) Markov random field models, 5) artificial neural networks, 6) classifiers, 7) deformable models and 8) atlas-guided approaches. Most of these approaches perform segmentation based on gray-level intensities; however, the gray-levels alone are not sufficient as many soft tissues in CT have overlapping gray-level ranges; thus, the use of higher-order properties of the corresponding anatomical structures is necessary to perform accurate medical image segmentation [4]. Further, many of these techniques perform either segmentation of a single object of interest, or segmentation of an entire image all at once. We propose a method to implement multiple single object segmentations (without using models) as a way of automatically segmenting multiple

Manuscript received April 24, 2006.

Jacob D. Furst is with DePaul University, Chicago, IL 60604 USA (phone: (312) 362-5158; fax: (312) 362-6116; e-mail: jfurst@ cti.depaul.edu).

Ruchaneewan Sosumboom is with DePaul University, Chicago, IL 60604 USA (e-mail: rsusombo@students.depaul.com).

Daniela S. Raicu is with DePaul University, Chicago, IL 60604 USA (email: draicu@cti.depaul.edu). objects of interest.

Since the shape of the same organ might also be different across a sequence of 2-D axial slices or, even more, across different patients, texture-based features have been proposed for segmentation as a way to quantify the homogeneity and consistency of soft tissues across multiple slices of a single organ. There are a large number of texture-based segmentation algorithms in the literature; among the most commonly used segmentation algorithms based on texture features are clustering techniques, region growing, and splitand-merge techniques. Segmentation using these traditional techniques requires considerable amounts of expert interactive guidance or does not incorporate any spatial modeling which can result in poor segmentation results.

We propose an approach for automatic multiple-organ segmentation in Computed Tomography (CT) data. The approach consists of applying, in parallel, single organ segmentations for each organ of interest. The single organ segmentations are each composed of three stages: first, a probability image of the organ of interest is obtained by applying a binary classification model obtained using pixelbased texture features (the organ of interest is one class and all the other pixels form the unknown class); second, since the classifier model does not incorporate any spatial information, an adaptive split-and-merge segmentation algorithm is applied on the organ probability image to overcome this drawback and remove the noise introduced by misclassified pixels; and third, the segmented organ boundaries determined at the end of the previous stage are iteratively refined using a region growing algorithm. At the end of all single organ segmentations, pixels containing more than one segment label are resolved based on a comparison of the region sizes and average probabilities over contested pixels.

\section{Single ORgan SEgMENTATION}

\section{A. Pixel-Level Texture Extraction}

Pixel-level texture extraction is the process of analyzing and quantifying the texture patterns within a small neighborhood around a pixel of interest. There are several texture models, including structural, transform methods, and statistical models [2]; among all of these models, it has been shown that the second-order statistical model (the co-occurrence matrix) produces better classification accuracy over the transform-based approach and structural method [3], [7]. The common technique used to extract texture information from the co-occurrence matrix model is based on the Haralick 
texture descriptors [8].

Since most of the soft tissues have overlapping gray-levels and the contrast among soft tissues is low within CT images, especially given the large range of gray-levels in DICOM format (4096 gray-levels/12-bit resolution is typical), we first preprocess the $\mathrm{CT}$ images using a clipped binning approach [6]; the binning approach will enhance the gray-level contrast among the soft tissues and further improve the texture features used in the classification step. Then, for the pixel of interest, a small neighborhood of size $N$ by $N$ is formed and the normalized co-occurrence matrix along with nine Haralick texture descriptors (entropy, energy, contrast, sum average, variance, correlation, maximum probability, inverse different moment, and cluster tendency) are calculated within the corresponding neighborhood for all possible displacements $(N-1)$ and four directions $\left(0^{\circ}, 45^{\circ}\right.$, $\left.90^{\circ}, 135^{\circ}\right)$. Since the nine descriptors will be calculated for each of the $(N-1) * 4$ co-occurrence matrices, there will be $(N$ $1)^{*} 4$ t9 texture descriptors which are not necessary independent of each other. In order to reduce the feature set and select the most important features, an information-gain criterion [10] is applied on the feature set. At the end of this stage, each pixel will be characterized by a p-dimensional feature vector $\left[f_{1}(d, \theta ; \gamma), \ldots, f_{p}(d, \theta ; \gamma)\right]$, where $p$ indicates the number of the most discriminating features, $d$ and $\theta$ represent the displacement and the angle, respectively, and $\gamma$ indicates the corresponding Haralick feature. The feature vector is further normalized by a min-max normalization technique [1] applied with respect to each individual feature.

For computational efficiency, the co-occurrence matrix implementation represents only the gray-levels that appear within the pixel neighborhood under consideration; this implementation is possible since the texture features are not affected by the co-occurrence probabilities equal to zero produced by the intensity pairs which do not appear in the corresponding pixel neighborhood. Therefore, rather than having a 256 by 256 co-occurrence matrix for each pixel neighborhood, the co-occurrence matrix will be, in the worst case scenario (when all possible values within the pixel neighborhood are different), of size $(2 N)^{2}$.

\section{B. Pixel-based Texture Classification}

In our approach, a binary Classification and Regression Tree (C\&RT) model is applied on the pixel-level data generated at the previous step in order to generate decision rules for the classification of the organ of interest. The C\&RT model is chosen because it does not make any assumptions about the distribution of the data, it is computationally efficient, it generates easily interpreted output data in the form of decision rules, and it has good classification performance [3]. Once the optimal decision tree is built, each terminal node will produce a decision rule for the organ of interest and a probability associated with each rule in the form of an IF (conditions on features)-THEN (label)-with PROBABILITY $=p$ expression; the probability $p$ will be given by the ratio between the number of true organ pixels classified as the organ of interest and total number of pixels found at that terminal node. For instance, a rule such as

$$
\operatorname{IF}\left[f_{1}(d, \theta ; \gamma)<0.34,0.65<f_{5}(d, \theta ; \gamma) \leq 0.8\right]
$$

THEN Liver with

PROBABILITY $=.9$

denotes a rule obtained from a terminal node in which $90 \%$ of the pixels found at that node where indeed liver pixels.

At the end of the classification process, the decision rules will be applied to each pixel within the slice to be segmented; the output will be an organ probability image used later in the adaptive split-and-merge segmentation stage.

\section{Adaptive Split \& Merge Segmentation}

Pixel-level classification is often an ultimate objective for image segmentation [5]. However, classifiers generally do not perform any spatial modeling and this makes the segmentation process sensitive to the noise produced by the misclassified pixels.

To overcome this limitation, we propose an adaptation of the split-and-merge algorithm that will be applied on the organ probability image. The algorithm involves two main steps: 1) the selection of initial seed regions and 2) the selection of adjacent regions.

The first step is done as indicated by SelectSeedRegion:

$$
\begin{aligned}
& \text { SelectSeedRegion }(R, \tau) \\
& \text { If } \mu_{\mathrm{P}(\mathrm{R})} \geq \tau \text {, then return } \mathrm{R} \\
& \text { Else if } \mathrm{R} \text { is bigger than } 16 \text { pixels by } 16 \text { pixels } \\
& \text { Divide } \mathrm{R} \text { into } 4 \text { quadrants } \mathrm{R}_{1}, \mathrm{R}_{2}, \mathrm{R}_{3}, \mathrm{R}_{4} \\
& \text { Return } \chi_{\mathrm{i}=1 \text { to } 4} \text { SelectSeedRegion }\left(R_{i}, \tau\right)
\end{aligned}
$$

In which $\mathrm{R}$ is a region of interest, $\mu_{\mathrm{P}(\mathrm{R})}$ is the average probability of the region $\mathrm{R}$ and $\tau$ is a probability threshold. The seed set $\mathrm{S}=$ SelectSeedRegion(I, 90\%), in which I is the entire probability image.

The second step is done as indicated by SelectAdjacentRegion:

\section{SelectAdjacentRegion $(R, \tau, S)$}

If $\mu_{\mathrm{P}(\mathrm{R})} \geq \tau$ and $\mathrm{R}$ is adjacent to $\mathrm{S}$, then return $\mathrm{R}$

Else if $\mathrm{R}$ is bigger than 4 pixels by 4 pixels

Divide $\mathrm{R}$ into 4 quadrants $\mathrm{R}_{1}, \mathrm{R}_{2}, \mathrm{R}_{3}, \mathrm{R}_{4}$

$$
\text { Return } \chi_{\mathrm{i}=1 \text { to } 4} \text { SelectAdjacentRegion }\left(R_{i}, \tau, S\right)
$$

The completion of the split and merge is $\mathrm{S} \chi$ SelectAdjacentRegion $(R, 80 \%, S)$ for all the 16 pixel by 16 pixel regions $\mathrm{R}$ adjacent to $\mathrm{S}$.

First, the image is split into smaller regions until all the regions meet a homogeneity criterion set by the threshold $\tau$ for splitting or the region size is smaller than a certain threshold. The homogeneity criterion is based on the 
average of the organ probabilities within the region considered for splitting; if the average probability is smaller than the threshold, the region is split further unless its size is smaller than a certain value. Second, the small split organ regions are grouped to form the organ of interest if they are adjacent to each other. In other words, the image is segmented into disjoint regions that correspond to the two classes, the organ of interest class and the unknown class (all the other tissues).

The threshold for splitting is a very important parameter. Its value will be set very high in order to find a reliable seed set (pixels close to the center of the organ region are expected to have high organ probabilities). Therefore, we allow the threshold to vary only at $90 \%$ and set the minimum region size of $16 \times 16$ in order to attain reliable.

Once the seed set is determined, the splitting constraints are relaxed. The process looks only at the regions adjacent to the seed set and splits these regions further if the two relaxed splitting criteria (decreased probability threshold and a new minimum sub-region size) are not satisfied. Again, the homogeneity threshold is relaxed down to $80 \%$ confidence since a lower level will introduce non-organ pixels within the segmented region. The cut off value for the splitting threshold is chosen automatically based on the sensitivity of the classifier with respect to the organ of interest. This potentially early termination (because of generally high sensitivities) may cause the system not to include some organ boundary and so a refinement stage is necessary as described in the following section.

\section{Adaptive Region Growing}

To refine the boundary of the segmented organ, an adaptive region growing approach is deployed as a postprocessing step. First, the process finds the edge-pixels of each segmented region. Around each edge-pixel, a window of size $N$ by $N$ is formed and the average probability within that window is calculated. The edge-pixel will be merged with the segmented region only if it satisfies the region constraint. The threshold for refinement is more relaxed compared with those used in the process of splitting since, at the organ boundary, the average of the organ probabilities tends to be lower. We set the threshold at $60 \%$ (lower than this will allow results obtained by chance) and the process repeats until no new pixel is added in the result of the region segmentation.

\section{Multiple Organ Segmentation}

Once the individual organ segmentations have been generated, they must be combined to generate the final segmentation showing all the segmented organs in the same image.

Each of the pixels in the set of single-organ images is compared and a final decision made about the segment to which it belongs. There are a number of possible cases, and the decision of which label to apply may depend on information beyond the pixel itself.

In the case of a pixel which has not been classified (each single-organ segmentation classified that pixel as "unknown") the pixel remains unclassified in the final segmentation. This may be a result of a pixel that is of no interest to the application, or it may be the result of not having a complete set of single-organ segmentation filters. In the latter case, the segmentation may be updated later by a subsequent single-organ segmentation and a new application of the multiple-organ resolution step.

In the case of a pixel which has been only labeled in one instance of a single-organ segmentation, that pixel retains its label in the final segmentation. Note that as further singleorgan segmentation filters are defined and applied, this pixel may be contested, in which case the steps below would be required.

In the final case, in which a pixel has multiple labels, the algorithms will make a decision on a single label for that pixel based on information about the region to which the pixel belongs. In particular, both the size of the region containing that pixel and the average probability of the labels for each pixel in the region will contribute to the final pixel label. In practice, this allows us to consider a 4-connected set of contested pixels as a group when deciding on which label to assign. Size is the more important of the two considerations: noise in the decision tree classifier may produce entire regions of misclassified pixels, but, in general, they will be smaller than regions generated by correct filters. In the cases where the regions are approximately equal in size (we have chosen a threshold of the larger being no greater than $10 \%$ the size of the smaller), the average probability of the regions are compared, and the label associated with the greatest average becomes the label for the contested pixels.

\section{PRELIMINARY RESUlts}

Our preliminary results are based on data extracted from normal CT studies from Northwestern Memorial Hospital $(\mathrm{NMH})$ PACS. The data consists of multiple, serial, axial computed tomography images derived from helical, multidetector $\mathrm{CT}$ abdominal and chest acquisitions using a HiSpeed CT/i scanner (GE Medical Systems, Milwaukee, WI). The images were transferred via Ethernet to a nearby computer workstation in DICOM format of size 512 by 512 and having 12-bit gray level resolution.

In order to obtain the prior knowledge for C\&RT, we randomly select pixels from one $\mathrm{CT}$ slide in which the organ of interest was present such that $50 \%$ of total pixels are from the organ of interest class and another $50 \%$ of total selected pixels are from "unknown" class. For each pixel, a 9x9 window is obtained to extract the pixel-level texture. In order to evaluate the model, the data are randomly divided into $66 \%$ for training and $34 \%$ for testing. The optimal C\&RT was found at approximately $88 \%$ sensitivity and over $96 \%$ for the specificity on the testing set. The classification rules 
were applied on a sequence of consecutive slices in which the interested organ was present.

Figure 1 (a)-(c) shows the results of individual organ segmentations that have been obtained through the split-andmerge algorithm, where the red color represents the higher threshold and the other colors represent lower probabilities as moving away from the core of the organ. Then, the single organ segmentation results are combined (as explained in Section III) generating the multiple organ segmentation shown in Figure 1(d). Figure 2 shows two examples of multiple organs segmentation results overlaid in the original 2-D slices.

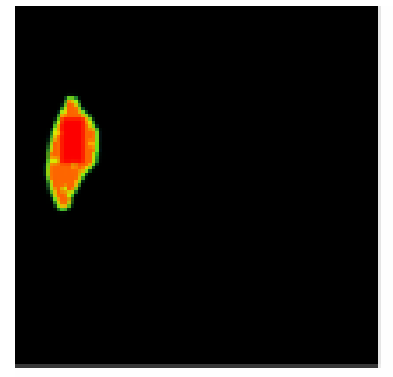

(a)

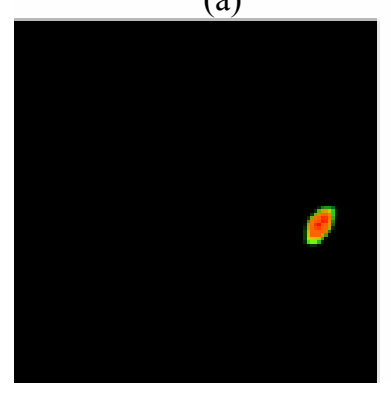

(c)

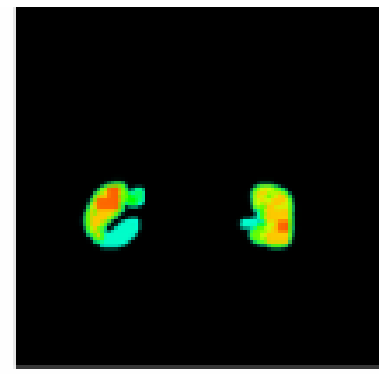

(b)

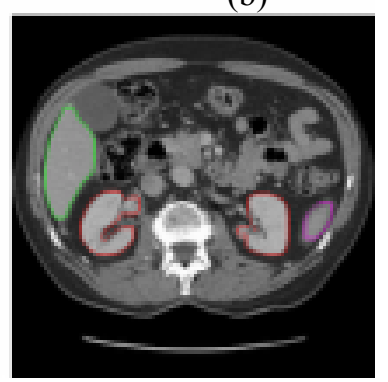

(d)
Figure 1: Example of multiple organ segmentation; (a) presents liver segmentation (b) presents kidney segmentation (c) presents spleen segmentation, and (e) is the final of multiple organ segmentation in which combining from 3 single organ filters
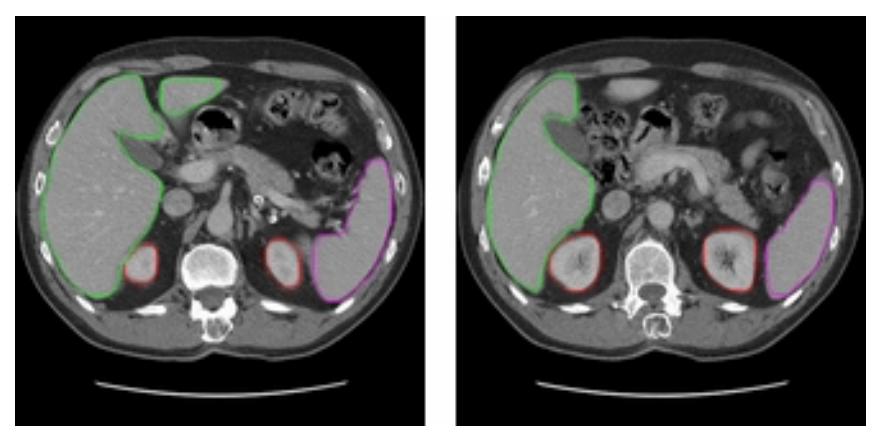

Figure 2: Example of multiple organ segmentation

\section{CONCLUSION}

The use of multiple, single organ segmentation filters provides a number of important benefits: 1) segmentation is no longer an "all or nothing" approach in which every pixel in the image must be applied a segment label; and 2) segmentations can be iteratively improved as information on new organs becomes available to generate new segmentation filters.

This work could be extended in a number of ways, including moving to three dimensional (3D) texture extraction and 3D segmentation algorithms. It could also be used to generate hierarchical segmentations supported by ontological labels in which segmentation filters are generated for subcomponents of existing segmentation filters based on an ontology defined for the particular domain or pathology of interest.

\section{REFERENCES}

[1] Channin, D., Raicu, D. S., Furst, J. D., Xu, D. H., Lilly, L., Limpsangsri, C.: Classification of Tissues in Computed Tomography using Decision Trees. Poster and Demo, The 90th Scientific Assembly and Annual Meeting of the Radiology Society of North America (RSNA04), 2004.

[2] Chang, K.I., Bowyer, K.W., Sivagurunath, M.: Evaluation of texture segmentation algorithms. IEEE Conf. on Computer Vision and Pattern Recognition, (1999) 294-299.

[3] Varma, M., Zisserman, A.: Texture classification: are filter banks necessary? In Proc. IEEE Conference on Computer Vision and Pattern Recognition, Vol. 2, (2003) 691-8.

[4] Dave, R. N., Fu, T.: Robust shape detection using Fuzzy clustering. Practical application Fuzzy Sets Syst., Vol. 65, (1994) 161-185.

[5] Pham, D.L., Xu, C., Prince, J.L.: Current methods in medical image segmentation. Annual Review of Biomedical Engineering, Vol. 2, (2000) 315-337.

[6] Lerman, R., Raicu, D.S., Furst, J.D.: Contrast enhancement of softtissues in Computed Tomography images. Proceedings of SPIE Medical Imaging Conference, 2006.

[7] Weszka, J., Deya, C., Rosenfeld, A.: A Comparative Study of Texture Measures for Terrain Classification. IEEE Trans. System, Man and Cybernetics, Vol. 6, (1976 ) 269-285.

[8] Haralick, R.M., Shanmugam, K., Dinstein, I.: Textural Features for Image Classification. IEEE Trans. on Systems, Man, and Cybernetics, Vol. Smc-3, no.6, (1973) 610-621.

[9] Olabarriaga, S.D., Smeulders, A.W.M.: Interaction in the segmentation in medical images: A survey. Medical Image Analysis, Vol. 5, No. 2, (2001) 127-142.

[10] Sonka, M., Hlavac, V., Boyle, R.: Image Processing, Analysis, and Machine Vision. Pacific Grove: Brooks/Cole Publishing Company, 1999. 